\title{
Investigating how explicit contextual cues affect predictive sensorimotor control in autistic adults.
}

\author{
Tom Arthur ${ }^{1,2}$, Mark Brosnan², David Harris', Gavin Buckingham, \\ Mark Wilson ${ }^{1}$, Genevieve Williams ${ }^{1}$ and Sam Vine ${ }^{1}$
}

1. Department of Sport and Health Sciences, College of Life and Environmental Sciences, University of Exeter, Exeter, EX1 2LU, UK.

2. Centre for Applied Autism Research, Department of Psychology, University of Bath, Bath, BA2 7AY, UK.

\begin{abstract}
Research suggests that sensorimotor difficulties in autism could be reduced by providing individuals with explicit contextual information. To test this, we examined autistic visuomotor control during a virtual racquetball task, in which participants hit normal and unexpectedly-bouncy balls using a handheld controller. The probability of facing each type of ball was varied unpredictably over time; however, during cued trials, participants received explicit information about the likelihood of facing each uncertain outcome. When compared to neurotypical controls, autistic individuals displayed poorer task performance, atypical gaze profiles, and more restricted swing kinematics. These visuomotor patterns were not significantly affected by contextual cues, indicating that autistic people exhibit underlying differences in how prior information and environmental uncertainty are dynamically modulated during movement tasks.
\end{abstract}

Key Words: autism, prediction, virtual-reality, active inference, uncertainty, volatility.

Acknowledgements: The authors would like to thank all of the participants who took part in this study. We would also like to thank the National Autistic Society for assistance with participant recruitment.

Corresponding Authors: Tom Arthur \& Dr Sam Vine

Department of Sport and Health Sciences,

College of Life and Environmental Sciences

University of Exeter, St Luke's Campus

Heavitree Road

Exeter, Devon, United Kingdom

EX1 2LU

Email: S.J.Vine@exeter.ac.uk

Tel: +44 1392722892 


\section{Introduction}

Individuals with Autism Spectrum Disorder (ASD; hereafter "autistic people"; Kenny et al., 2016) can face a range of sensorimotor difficulties, such as clumsiness, sensory disturbances, poor postural control, and issues with eye-hand coordination (see Fournier et al., 2010; Gowen \& Hamilton, 2013; Coll et al., 2020 for reviews). These difficulties may be associated with restricted personal independence (Jasmin et al., 2009), deleterious health outcomes (McCoy et al., 2016), and atypical sociobehavioural development (Sutera et al., 2007; MacDonald et al., 2013). However, there is a lack of evidence-based interventions that are proven to effectively tackle autismrelated sensorimotor issues and their underlying causes (Colombo-Dougovito \& Block, 2019). The present research aims to initiate this line of enquiry, by exploring whether movement-based skills in autistic people are enhanced following the provision of explicit prior information about an individual's current sensory environment.

A growing body of research suggests that sensorimotor interventions in autism should be focusing on aspects of predictive control. Autism-related movement atypicalities often reside in the planning or anticipatory stages of an action (Hughes, 1996; Schmitz et al., 2003; Fabbri-Destro et al., 2009; Cannon et al., 2021; Cavallo et al., 2021) and appear underpinned by context-sensitive differences in the use of prior expectations (Palmer, Paton, et al., 2015; Arthur et al., 2020; 2021). Relatedly, autistic participants display atypical responses to unexpected sensory cues, while coordinating visuomotor actions as if their surroundings are highly unpredictable or volatile (Lawson et al., 2017; Arthur et al., 2021). This suggests that autistic people may have difficulties forming stable predictions about their environment (Lawson et al., 2014; Van de Cruys et al., 2014). Indeed, predictive processing theories propose that higher-level beliefs (about uncertainty and stability) play a crucial role in regulating the dynamic weighting of topdown predictions and bottom-up sensory information (Yu \& Dayan, 2003; Mathys et al., 2014). Given the increasing evidence that these mechanisms are atypical in autism (Palmer et al., 2017), interventions should look to help autistic people build flexible, contextually-appropriate predictions that can guide their sensorimotor actions.

A promising method for enhancing predictive sensorimotor control is to provide prior contextual information about the uncertain (and often complex) world that surrounds a task (see Haker et al., 2016). The notion of making conditions 'more understandable' 
is commonly advocated in the field, and studies show that autistic people can be explicitly cued or primed to process context in social cognition and perceptual discrimination tasks (e.g., Plaisted et al., 1999; López et al., 2004; Balconi et al., 2012; Vermeulen, 2015; Gowen et al., 2020; Cannon et al., 2021). From a mechanistic viewpoint, such an approach could particularly help individuals that have difficulties extracting the ambiguous contextual relationships that underpin dynamic sensorimotor interactions (e.g., autistic people: Qian \& Lipkin, 2011; Van de Cruys et al., 2014). Indeed, a person's ability to detect implicit and probabilistic regularities from the world around them can shape their higher-level 'meta-cognitive' beliefs about environmental uncertainty and stability (see Yon \& Frith, 2021). So, by providing dynamic and explicit information about a task's underlying probabilistic state, one may be able to enhance the context-sensitive modelling of sensorimotor predictions in this setting.

Nevertheless, the degree to which explicit contextual cues can augment autistic sensorimotor abilities remains unclear. There are a lack of studies examining autistic movement control within naturalistic tasks, and it is uncertain whether such difficulties reflect impairments in making accurate predictions (as suggested above) or differences in modulating actions according to predictions about world and body states. Crucially, the weighting of sensory information and prediction errors is dependent on an array of neuromodulatory functions that may be atypical in autism (i.e., physiological systems that regulate synaptic gain signalling across hierarchical neural networks; Lawson et al., 2014; Quattrocki \& Friston, 2014; Van de Cruys et al., 2014). These include: phasic noradrenergic activity (Yu \& Dayan, 2003; Lawson et al., 2021), dopamine-serotonin interactions (Friston et al., 2012), and signalling in the anterior cingulate cortex and cerebellum (Behrens et al., 2007; den Ouden et al., 2010; Palacios et al., 2021). Differences in these systems could lead to pathological neural gain and disproportionate receptiveness to sensory inputs (Lawson et al., 2014), irrespective of any explicit contextual aids. Indeed, it has been found that some prediction-related atypicalities in autism persist even after individuals have been told about likely upcoming events (Thillay et al., 2016; Balsters et al., 2017; Greene et al., 2019).

Likewise, sensorimotor differences may relate to inherent psycho-behavioural traits that are largely stable and unreceptive to contextual cues. For instance, intolerance of uncertainty has been widely documented in autistic populations (Boulter et al., 2014; Vasa et al., 2018). Individuals with greater intolerance of uncertainty often experience 
adverse emotional reactions to unpredictable stimuli (Dugas et al., 1997). Though mechanistically distinct from higher-level state estimates (see Bervoets et al., 2021), recent data suggest that associated increases in anxiety could impair key predictive processing functions (e.g. volatility-related learning rate modulation, Lawson et al., 2021). Furthermore, sensory issues in autism correlate with apprehension about environmental change (Wigham et al., 2015; Pickard et al., 2020). Therefore, the degree to which autistic people benefit from explicit contextual information could also depend on trait differences in behavioural inflexibility and/or intolerance of uncertainty.

\section{The Present Research}

This study examined the effects of explicit probabilistic cues on autistic sensorimotor control. Specifically, prior contextual cues were provided in Virtual-Reality (VR) to help individuals make accurate, context-sensitive predictions during interceptive motor actions (Figure 1). Research has shown that neurotypical individuals significantly benefit from this type of information during action-based tasks (Navia et al., 2013; Gray, 2015; Gredin et al., 2018). For instance, football goalkeepers display enhanced performance when provided with advanced information about an opponent's most likely shooting direction (Navia et al., 2013). Although this type of intervention has not yet been tested in clinical populations, adaptive effects have been observed in autistic visual processing and motor imitation abilities when participants are explicitly cued towards goal-relevant contextual stimuli (López et al., 2004; Fulceri et al., 2018; Gowen et al., 2020; Soroor et al., 2021). Based on this evidence (and the studies discussed in the section above), we hypothesised that autistic people would show superior interceptive motor performances under cued versus non-cued trial conditions.

To further study the effects of explicit contextual cues on sensorimotor behaviours, we focused on participants' hand movements and visual sampling responses. During interceptive tasks, a person's movement patterns and gaze behaviours will be highly influenced by their prior expectations (see Mann, 2019; Arthur \& Harris, 2021). For instance, when an approaching ball is about to bounce, participants will direct their gaze away from its existing location towards its expected future position (Diaz et al., 2013; Mann et al., 2019), in what is referred to hereafter as the predictive bounce fixation. These behaviours are then updated in a context-sensitive manner. So, if ball elasticity (i.e., bounciness) is unexpectedly high and changeable, participants tend to 
increase the height and variability of their predictive bounce fixations, while reducing range of motion (ROM) in their swing (Arthur \& Harris, 2021). Although our previous work (Arthur et al., 2021) illustrated that these predictive visuomotor adjustments are exhibited in both autistic and non-autistic populations, we observed that autistic people generally show higher, more variable fixations and more restricted swing movements than their neurotypical counterparts. These data imply that beliefs about environmental uncertainty and/or volatility are atypically high in these individuals. However, the explicit contextual cues in this study were designed to augment trial-by-trial state predictions, while reducing estimates of environmental uncertainty. Accordingly, we hypothesised that swing ROM would increase, and the location of predictive bounce fixations would decrease, in autistic participants from non-cued to cued conditions.

(Insert Figure 1 here)

\begin{abstract}
Methods
We employed a virtual racquetball paradigm which has previously be used to investigate context-sensitive predictive action responses in neurotypical and autistic populations (Diaz et al., 2013; Arthur \& Harris, 2021; Arthur et al., 2021). In this task, participants use a VR handheld controller to intercept balls that either have normal or unexpectedly-high levels of bounciness. Environmental uncertainty and volatility are then systematically manipulated, through varying the likelihood of facing a normal or bouncy ball over time (in a manner that is consistent with computational studies in the field; e.g., Lawson et al., 2017). Our previous research has observed that autistic participants exhibit impaired sensorimotor performances in this task, especially under more volatile probabilistic conditions (Arthur et al., 2021). However, the present study provided participants with prior information about the probability of facing a normal or bouncy ball over time. This would permit examination of whether sensorimotor control can be enhanced in autistic people through the use of explicit contextual cues.
\end{abstract}




\section{Participants}

A total of 44 participants took part in the study (30 male, 14 female, 40 right-handed, mean age: $29 \pm 7$ years). 22 individuals had a formal diagnosis of ASD, as provided by an expert clinician according to DSM-IV (American Psychiatric Association, 2013) or ICD-10 (World Health Organisation, 2012) criteria, while the remaining sample were age- and gender-matched neurotypical controls. Though analysis was primarily interested in assessing cue-related changes in the ASD group, these neurotypical individuals could help elucidate any autism-related atypicalities shown between conditions. Inclusion criteria specified that participants were to be at least sixteen years of age. Participants were excluded if they had any co-occurring medical conditions or learning disabilities that are known to affect sensorimotor control. Eligible participants were naïve to the study aims and had no previous experience of playing VR-based racquet sports. All individuals in the ASD group scored above the clinical 'screening cut-off' of 26 on the 50-item Autistic Quotient (AQ; Baron-Cohen et al., 2001), with clinical characteristics proving highly consistent with previously reported values (Table 1; for normative data, see Baron-Cohen et al., 2001; Woodbury-Smith et al., 2005). Informed consent was obtained ahead of all study procedures, in accordance with British Psychological Society guidelines. The study received approval from the Department of Sport and Health Sciences Ethics Committee (University of Exeter, UK) and the Department of Psychology Ethics Committee (University of Bath, UK).

(Insert Table 1 here)

\section{Materials}

The virtual racquetball environment was developed on the gaming engine Unity (Unity Technologies, San Francisco, CA) and is described at length in Arthur et al. (2021). It was presented to participants on an HTC VivePro head-mounted display at $120 \mathrm{~Hz}$ (HTC Inc., Taoyuan City, Taiwan). This consumer-grade, high-precision VR system comprises two 'lighthouse' base stations, which record movements of the headset and hand controller at $90 \mathrm{~Hz}$. The headset also contains an inbuilt eye-tracking system, 
which monitors user's gaze at $120 \mathrm{~Hz}$ with a spatial accuracy of 0.5-1.1. Participants were presented with a simulated $15 \times 15 \mathrm{~m}$ racquetball court, which contained a circular target on its front wall (Figure 1A). They were required to position themselves $9 \mathrm{~m}$ behind this location before attempting to hit virtual balls towards the middle of the target using a VR hand controller. This controller was displayed as a $0.6 \times 0.3 \times 0.01 \mathrm{~m}$ virtual racquet, and the balls resembled the appearance and size of those in 'real-world' tennis (Figure 1A). All balls were launched from a height of $2 \mathrm{~m}$, following three auditory tones, and would bounce $3.5 \mathrm{~m}$ in front of participant's prescribed starting position. Their trajectory passed through the midline of the room, which was $0.75 \mathrm{~m}$ to the right (for right-handers) or left (for left-handers) of this predetermined starting position.

For this study, the virtual environment was further adapted for the cued experimental condition. In these trials, participants would transition between five game 'levels', which provided explicit information about ball bounciness and environmental probabilities (Figure 1). Level changes were signalled by an auditory tone and brief 'loading screen' (see Supplementary Video at: https://osf.io/5y48g/). Following this transition, participants would be transported into a new virtual room, to signal that their surrounding environment had changed. The front wall was visually identical for all levels, as were any ball bounciness- or goal-related action cues (e.g., the ball, floor, target and racquet). However, to emphasise that the underlying contextual probabilities had changed with each level transition, participants were presented with visual 'hawkeye' cues immediately after the loading screen (Figure 1C). These illustrations projected the upcoming trajectory and ratio of normal and bouncy balls in each game level. Such 'hawkeye' cues were presented for 10 seconds and accurately represented the probabilistic 'ground truth' of a given level. They were accompanied by a visual indicator (referred to as the 'bounceometer'), which explicitly stated whether the likelihood of getting a bouncy ball was low, medium, or high (Figure 1D). Although this probabilistic information only reflected the statistical structure of a given level (i.e., they were not varied on trial-by-trial basis), they were presented for $3 \mathrm{~s}$ ahead of each trial in the cued condition. Such advanced information was not available in the practice or control conditions, nor in the final nine trials (i.e., game level) of the cued block.

Participants also completed the AQ (Baron-Cohen et al., 2001) and Intolerance of Uncertainty Scale-shortened version (IUS-S; Carleton et al., 2007). The AQ indexes five autistic-like traits: communication, imagination, social skills, attention switching, 
and attention to detail. Each trait subscale is scored out of ten and combined into an overall total (possible range: 0-50), which is said to index where an individual is situated on the 'autism spectrum' (Baron-Cohen et al., 2001). The resulting scores are continuous and normally-distributed in general populations, with clinical ASD viewed to reside at the extreme higher end of this continuum (Baron-Cohen et al., 2001; Ruzich et al., 2015). Conversely, the IUS-S is a 12-item questionnaire measuring intolerance of uncertainty, defined as the tendency of an individual to consider the possibility of a negative event occurring unacceptable, irrespective of the probability of occurrence (Carleton et al., 2007). Itemised statements are rated from 1 (not at all characteristic of me) to 5 (entirely characteristic of me) and combined into a total out of 60 . Higher scores reflect greater intolerance of uncertainty, as is commonly reported in autistic populations (Boulter et al., 2014; Wigham et al., 2015; Pickard et al., 2020).

\section{Procedures}

After providing written informed consent, participants were fitted with the headmounted display and familiarised with the virtual environment. At this stage, the eyetracker was calibrated over five gaze locations using the manufacturer's built-in routine. Calibration was repeated before each experimental condition and upon any obvious displacement of the VR headset. Once familiarised with the virtual environment, participants then completed thirty baseline racquetball trials. Throughout this initial block, all virtual balls followed the same pre- and post-bounce trajectory, which were consistent with the effects of gravity $\left(-9.8 \mathrm{~m} / \mathrm{s}^{2}\right)$. Their speed remained fixed at $-9 \mathrm{~m} / \mathrm{s}$ in the vertical plane (at the time of bounce), and elasticity was set at standard tennis ball levels $(65 \%)$. Participants were instructed to hit balls towards the centre of the target, but that they would not be able to see or feel where they go after hitting them. This lack of feedback was used in all experimental trials to minimise confounding effects relating to motivation, communication skills, and task reward/error. Instead, a neutral 'pop' sound signalled when balls had made contact with the racquet, and any subsequent auditory and visual ball information was removed after this event.

Following the initial baseline trials, participants performed two counterbalanced experimental conditions. In both of these blocks, ball bounciness was systematically varied over time to create unstable trial order sequences (illustrated in Figure 2). While two-thirds of trials would contain 'normal' balls that were the same as those faced at 
baseline (and in real-world environments), a third contained 'bouncy' balls with unexpectedly high levels of elasticity (85\%). This discernible change in post-bounce ball trajectory occurred without participant's knowledge and would likely deviate away from any prior experiences obtained during 'real-world' actions. The pre-bounce ball speeds and trajectories were always the same as in baseline, meaning that the different type of balls were impossible to tell apart until they had made contact with the floor. Importantly, the probability of facing a normal ball changed every 6, 9 or 12 trials (between $83 \%, 67 \%$ and $50 \%$ likely). These unpredictably changeable post-bounce ball trajectories created a volatile environment, in which autistic people display impaired interceptive performances (Arthur et al., 2021).

(Insert Figure 2 here)

Participants were randomly allocated one of three possible trial order sequences (available at https://osf.io/5y48g/) which would be presented to them in both experimental conditions. For the control block, individuals did not receive any probabilistic information about likely ball bounciness and trials were presented as one continuous sequence (Figure 2A). Instead, they were simply told that some balls may be more bouncy than others and that they should aim to hit as many of them as possible to the middle of the target.

Conversely, explicit information about situational probabilities were provided in the cued block. Here, visual cues indicated to participants both when ball bounciness probabilities were switching and how likely they were to face a 'normal' or 'bouncy' ball at any given time (see Figure 2B). This direct provision of contextual priors has been proven to enhance visuomotor control in various neurotypical performance domains (e.g., Navia et al., 2013; Gray, 2015; Gredin et al., 2018). Participants were told that these visual cues would help show them where the balls are going to go and how likely they are to get a bouncy ball during each game level. They were not informed that the trial order sequences would be exactly the same in each experimental block. 
The laboratory protocol generally lasted $\sim 30$ minutes in total. The two experimental conditions contained 45 trials each and were separated by a short break. The final 9 trials of each block contained identical visual information (i.e., no 'hawkeye' or 'bounceometer' cues) and thus provided a set of order-matched 'catch' trials for further examination (see Figure 2).

\section{Data Analysis}

Task performance was evaluated based on interception rate, which reflected the percentage of trials in which participants successfully hit the ball with their racquet. Kinematic variables were assessed using the positional data of the VR hand controller, which were extracted and then smoothed using a dual-pass, zero-phase Butterworth filter (frequency: $10 \mathrm{~Hz}$; Franks et al., 1990). Specifically, analysis focused on the foreswing phase of interceptive actions, which started when the racquet first began to move forward and ended when it first made contact with the ball. In trials where participants failed to hit the ball, foreswing offset represents the final data point in which the ball's depth position exceeded that of the racquet.

Peak velocity of the hand controller (in the direction of the target) was recorded from participants' foreswing movements, as autistic participants displayed slower, more novice-like swing actions than neurotypical individuals in our previous work (Arthur et al., 2021). ROM was assessed during this trial period to capture context-sensitive aspects of motor control. This outcome highlighted the maximum angular deviation between the VR headset and hand controller, as defined in the tranverse plane. Higher values would indicate that the hand had rotated to a greater degree around the body during the foreswing action. Conversely, decreases in ROM may signify that participants were 'fixing' movement degrees of freedom, a response which is typically prominent under volatile conditions and in autistic populations (Arthur et al., 2021).

Eye tracking data were converted into 'in-world' angular vectors, as defined according to head-centred egocentric coordinates. Yaw and pitch values were smoothed using a three-frame median filter and then a second-order Butterworth filter (at $15 \mathrm{~Hz}$; Cesqui et al., 2015). Since autistic participants employed anticipatory saccades in a similar manner to neurotypical individuals in our previous work (Arthur et al., 2021), analysis only focused on predictive fixations. To extract this information, cleaned data were 
entered into a spatial dispersion algorithm (Krassanakis et al., 2014), which identified periods where gaze remained steady within a $3^{\circ}$ area for a minimum of $100 \mathrm{~ms}$. Trials where eye-tracking was temporarily lost $(>100 \mathrm{~ms})$ or where there were $>20 \%$ of missing data were excluded. Subsequent analyses focused on the median onset time, mean duration, and average vertical position (mean pitch angle) of fixations that occur during (within $0.1 \mathrm{~s}$ ), or immediately prior to, ball bounces in each trial. This bounce fixation is elevated when an individual predicts that ball elasticity likely to be higher (Diaz et al., 2013; Mann et al., 2019). Furthermore, trial-to-trial variability in this bounce fixation location is typically increased under volatile conditions (Arthur \& Harris, 2021).

A-priori power calculations indicated that a sample size of 40 would be sufficient to detect any moderate statistical effects in this study $(f=.47)$, as estimated based on our previous data (Arthur et al., 2021) and data in Lawson et al. (2017). Such calculations were conducted using $\mathrm{G}^{*}$ Power 3.1 (Faul et al., 2007), with alpha set at $p=0.05$ and power $(1-\beta)$ at 0.80 . Poor motion tracking led to missing hand position data for one autistic participant. As such, they and their matched neurotypical counterpart were excluded from kinematic analyses (remaining $n=42$ ). A separate pair of matched cases were removed from gaze analyses, due to frequent loss of eye-tracking signal (remaining $n=42$ ). Data were deemed missing completely at random (Little's MCAR test: $p>0.05)$. Two further autistic participants were identified as potential outliers in the interception rate data, but their average scores (45.56\%, 54.44\%) were consistent with previous values and the overall pattern of results was not affected by their inclusion. In these instances, conventions recommend that extreme values are not removed (Aguinis et al., 2013), as case exclusion may disregard important information relating to clinical difficulties. Consequently, no data were excluded for this variable.

All variables were entered into separate mixed-model ANOVAs, which studied main effects of condition (cued vs control) and group (ASD vs neurotypical), as well as group-by-condition interactions. Effect sizes were quantified using partial-eta squared. Significant effects were followed up using post-hoc $t$-tests, which were adjusted using the Bonferroni correction. Prior to running these mixed-model ANOVAs, manipulation checks examined whether predictive bounce fixations were sensitive to dynamic environmental probabilities. Here, dependent $t$-tests examined changes in bounce fixation pitch angles between baseline and control trials, to see whether unexpected and volatile manipulations of ball bounciness led to significant adjustments in predictive 
gaze positions. To explore relationships with autistic-like traits and intolerance of uncertainty, Pearson's Correlation analysis examined associations between $A Q$ scores, IUS-S scores, and all sensorimotor outcomes.

Interception rate data were positively skewed, with 10 participants intercepting $100 \%$ of balls. This outcome deviated from normality, along with peak swing velocity, fixation onset time, and fixation duration (all $p<.05$ for Shapiro-Wilk test). Mixed-model ANOVAs are robust to moderate deviations from statistical normality (Lix et al., 1996) and were still performed. However, Mann-Whitney $U$ tests were employed for followup comparisons (using the Bonferroni correction) and Spearman's Rho for assessing their correlations with $A Q$ and IUS-S scores. Levene's Test highlighted significantly different levels of variance for bounce fixation pitch measures $(p<.05)$. No further assumptions were violated in relation to normality, sphericity, and homogeneity of variance. All statistical tests were conducted with alpha set at $p<.05$ and are reported alongside a Bayes Factor computation, which illustrates the strength of evidence in favour of the alternative/null hypotheses. Statistical procedures were undertaken using JASP 0.12.2, with the full dataset available at https://osf.io/5y48g/.

\section{Results}

\section{Manipulation Checks}

Manipulation checks showed a significant change in the height of predictive bounce fixations between baseline and control conditions (average pitch angle: $t(39)=6.73, p$ $\left.<.001, \mathrm{BF}_{10}=2.76 \times 10^{5}\right)$. As expected, volatile fluctuations in ball bounciness caused both groups to cast their gaze at a higher spatial location than at baseline, despite their being no explicit informational cues in either block of trials. These results confirm assumptions that participants would elevate their predictive bounce fixations when faced with unexpectedly bouncy balls and volatile trial conditions.

\section{Task Performance and Swing Kinematics}

For task performance, analysis revealed a significant main effect of group $(F(1,42)=$ 8.44, $\left.p=.01, \eta_{p}^{2}=0.17, \mathrm{BF}_{10}=7.41\right)$, with average interception rates significantly lower in autistic $(87.75 \pm 14.78 \%)$ compared to neurotypical participants (97.22 \pm 
$3.91 \% ; W=379.50, p=.001, \mathrm{BF}_{10}=13.23$; Figure 3). However, there were no significant condition effects $\left(F(1,42)=.08, p=.78, \eta_{p}^{2}<0.01, \mathrm{BF}_{10}=0.23\right)$ or groupby-condition interactions $\left(F(1,42)=.06, p=.81, \eta_{p}^{2}=0.001, \mathrm{BF}_{10}=0.28\right)$. AQ scores negatively correlated with interception rate in both control $\left(R s=-.34, p=.02, \mathrm{BF}_{10}=\right.$ $2.69)$ and cued $\left(R s=-.41, p=.01, \mathrm{BF}_{10}=13.67\right)$ conditions. Conversely, IUS-S values were not significantly associated with task performance in either block of trials (control: $R s=-.16, p=.42, \mathrm{BF}_{10}=0.28 ;$ cued: $\left.R s=-.17, p=.26, \mathrm{BF}_{10}=0.41\right)$.

(Insert Figure 3 here)

There were no significant main effects of group $\left(F(1,40)=1.47, p=.23, \eta_{p}^{2}=.0 .04\right.$, $\left.\mathrm{BF}_{10}=0.70\right)$ or condition $\left(F(1,40)=0.13, p=.73, \eta_{p}^{2}<.01, \mathrm{BF}_{10}=0.23\right)$ for peak swing velocity, nor were there any significant interactions for this metric $(F(1,40)=3.82, p=$ $\left..06, \eta_{p}^{2}=.09, \mathrm{BF}_{10}=0.97\right)$. In terms of ROM, there was a main effect of group $(F(1,40)$ $\left.=7.58, p=.01, \eta_{p}^{2}=0.16, \mathrm{BF}_{10}=4.43\right)$ and a significant group-by-condition interaction $\left(F(1,40)=8.88, p=.01, \eta_{p}^{2}=0.18, \mathrm{BF}_{10}=7.50\right)$. Autistic participants exhibited lower ROM than neurotypical participants (see Figure 4). However, while these individuals generally decreased ROM between control and cued conditions (Mean difference: $6.50 \pm 12.38^{\circ} ; t(20)=2.41, p=.03, \mathrm{BF}_{10}=2.30$ ), neurotypical values remained relatively stable (Mean difference: $5.50 \pm 13.68^{\circ} ; t(20)=1.84, p=.08, \mathrm{BF}_{10}=0.95$ ). $\mathrm{ROM}$ significantly correlated with $\mathrm{AQ}$ scores in the cued $\left(R=-.39, p=.01, \mathrm{BF}_{10}=4.38\right)$ but not the control trials $\left(R=-.25, p=.11, \mathrm{BF}_{10}=0.66\right)$. Moreover, $\mathrm{ROM}$ negatively associated with IUS-S scores in both conditions (control: $R=-.42, p=.01, \mathrm{BF}_{10}=8.40$; cued: $R=-.42, p=.01, \mathrm{BF}_{10}=7.32$ ). Peak swing velocities did not significantly correlate with $\mathrm{AQ}$ or IUS-S scores during either condition ( $p$ 's $>.06$, all $\mathrm{BF}_{10}<2$ ).

(Insert Figure 4 here) 


\section{Gaze Data}

Groups exhibited similar gaze profiles during the task. ANOVAs revealed no significant group differences or group-by-condition interactions in relation to the onset and duration of predictive bounce fixations ( $p$ 's $>.30$; all $\mathrm{BF}_{10}<0.67$ ). Participants maintained slightly longer fixations during the cued trials (Figure 5), with a significant effect of condition emerging for this metric $\left(F(1,40)=6.72, p=.01, \eta_{p}^{2}=0.14, \mathrm{BF}_{10}=\right.$ 3.49). However, this main effect did not emerge in relation to onset time $(F(1,40)=$ 1.56, $\left.p=.22, \eta_{p}^{2}=0.04, \mathrm{BF}_{10}=0.44\right)$, and there were no significant AQ or IUS-S correlations for either fixation metric ( $p$ 's > .18; all $\mathrm{BF}_{10}<0.67$ ).

(Insert Figure 5 here)

Next, the average pitch angle (i.e., vertical position) of participant's predictive bounce fixation was examined. Here, both a significant main effect of condition $(F(1,40)=6.39$, $\left.p=.02, \eta_{p}^{2}=0.14, \mathrm{BF}_{10}=2.10\right)$ and a significant group-by-condition interaction emerged $\left(F(1,40)=7.92, p=.01, \eta_{p}^{2}=0.17, \mathrm{BF}_{10}=5.32\right)$. Average pitch values generally increased from control to cued trials, however these changes were groupdependent (see Figure 5). Specifically, neurotypical participants elevated the height of their bounce fixations after receiving explicit probabilistic cues (Mean difference: 1.96 $\left.\pm 1.93^{\circ} ; t(20)=4.64, p<.001, \mathrm{BF}_{10}=184.36\right)$, whereas autistic participants showed minimal changes between blocks (Mean difference: $0.11 \pm 2.75^{\circ} ; t(20)=.18, p=.86$, $\mathrm{BF}_{10}=0.23$ ). Surprisingly though, no statistical relationships emerged between bounce fixation pitch angles and scores on the AQ or IUS-S ( $p$ 's > .19; all BF $10<0.50$ ).

Finally, the trial-to-trial variability in participant's predictive bounce fixation location was examined. This analysis revealed a significant main effect of group $(F(1,40)=6.99, p=$ $\left..01, \eta_{p}^{2}=0.15, \mathrm{BF}_{10}=4.63\right)$. Generally, autistic participants showed higher pitch angle SDs than their neurotypical counterparts (Figure 6). However, these variability scores did not significantly differ between conditions $\left(F(1,40)=1.69, p=.20, \eta_{p}^{2}=0.04, \mathrm{BF}_{10}\right.$ $=0.46)$, and there were no significant group-by-condition interactions $(F(1,40)=1.75$, $\left.p=.19, \eta_{p}^{2}=0.04, \mathrm{BF}_{10}=0.57\right)$. Furthermore, no statistical associations emerged between pitch angle SD, AQ totals, and IUS-S scores ( $p$ 's $>.12$, all BF $10<0.67$ ). 
(Insert Figure 6 here)

\section{Discussion}

This study examined the effects of explicit contextual cues on autistic sensorimotor behaviours during a VR-based interceptive racquetball task. Previous research has shown that prior situational information about likely task outcomes can enhance neurotypical action responses (Navia et al., 2013; Gray, 2015; Gredin et al., 2018), and it has been suggested that such an approach could help autistic individuals in uncertain sensory environments (Qian \& Lipkin, 2011; Haker et al., 2016). Therefore, it was hypothesised that autistic people would display improved task performances and enhanced predictive sensorimotor control under explicit cue conditions in this study.

Contrary to these initial hypotheses, we found no significant effects of the explicit contextual cues on interceptive task performances. Lower interception rates and more restricted swing kinematics were evident in autistic sensorimotor responses (Figures 3-4), irrespective of any prior probabilistic information. Such impaired motor responses replicate previous findings in this task (Arthur et al., 2021) and in a host of other empirical assessments (e.g., Green et al., 2002; Whyatt \& Craig, 2013; Chen et al., 2019). Crucially though, results indicated that there were no generic difficulties in understanding the VR-based contextual cues in our study (see Supplementary Analyses). Indeed, it has already been demonstrated that autistic people can use explicit situational information to guide perceptual and motor abilities (Balconi et al., 2012; Vermeulen, 2015; Thillay et al., 2016; Fulceri et al., 2018; Gowen et al., 2020; Soroor et al., 2021). Instead, null findings suggest that autistic people simply did not benefit from the explicit contextual information that was afforded to them in this task.

Notably, the contextual cues in this study provided explicit and veridical information about environmental uncertainty, in a manner that was designed to enhance dynamic state predictions. However, contrary to our hypotheses, we found no evidence for enhanced predictive sensorimotor control under the cued experimental trials. Indeed, while neurotypical participants used the explicit contextual cues to adjust their gaze fixation behaviours (i.e., they directed them to higher spatial locations), autistic 
individuals showed minimal between-condition changes in their prediction-related visual sampling responses (Figure 5). Though surprising, these findings align with observations that certain prediction-related atypicalities in autism persist in the face of veridical visual cues about likely trial outcomes (Thillay et al., 2016; Balsters et al., 2017; Greene et al., 2019; Cannon et al., 2021). In fact, the autism group appeared to restrict swing ROM under cued conditions (Figure 4), a response which typically coincides with heightened uncertainty estimates (Arthur \& Harris, 2021). Results therefore imply that autistic people were over-reactive to both implicit and explicit cues about environmental volatility, causing them to employ visuomotor behaviours that are typically affiliated with imprecise (i.e., uncertain) higher-level beliefs.

Taken together, our findings support proposals that autistic sensorimotor control is underpinned by atypicalities in the dynamic modulation of prediction error (e.g., Friston et al., 2013; Lawson et al., 2014; Van de Cruys et al., 2014). Specifically, autistic people were able to detect environmental changes and learn implicit cue-outcome relationships (see also Manning et al., 2017; Sapey-Triomphe et al., 2021); however, their trial-by-trial weighting and updating of action predictions proved to be suboptimal. According to predictive coding perspectives, the context-sensitive mechanisms that regulate synaptic gain signalling (and thus, the weighting of top-down and bottom-up activity) may be atypical in autistic people, leading to a pathologically high receptiveness to sensory inputs and an over-reactivity to contextual change (see Lawson et al., 2017). Such proposals are consistent with our data, which imply that autistic people show a heightened responsivity to recent unexpected events. Indeed, compared to neurotypical individuals, autistic participants directed predictive bounce fixations towards higher spatial locations (Figure 4) and updated these behaviours more variably from trial to trial (Figure 6). These profiles reflect an increased tendency to prepare for probabilistically salient and/or volatile events, which directly replicates the findings of our previous work (Arthur et al., 2021) and in Lawson et al. (2017).

Nonetheless, there was substantial inter-individual variability observed in the dataset. Figures 3-6 illustrate diverging responsivity to contextual cues and levels of task performance, with such heterogeneity proving particularly prominent in autistic individuals. These wide-ranging data patterns are consistent with clinical research (Fournier et al., 2010; Coll et al., 2020), and suggest that sensorimotor difficulties may bear varied aetiologies and neurobiological underpinnings. This notion is not at odds 
with predictive processing frameworks, as synaptic gain control is theoretically underpinned by a myriad of interacting networks and modulatory systems (Lawson et al., 2014). Future work must consider these heterogeneous individual aetiologies when attempting to reduce sensorimotor difficulties through applied interventions.

Notably, relationships between sensorimotor control and intolerance of uncertainty were trivial in this study. IUS-S scores did not significantly correlate with any of our performance or visual sampling metrics. These null effects are perhaps unsurprising, as associations between intolerance of uncertainty and anxiety are mechanistically distinct from those concerning hierarchical state estimates and predictions (Bervoets et al., 2021). Indeed, the IUS-S indexes an individual's chronic disposition to appraise uncertain outcomes as aversive (Carleton et al., 2007), which sits in stark contrast to the highly dynamic and context-sensitive predictive behaviours that were assessed in our virtual racquetball task. Nevertheless, intolerance of uncertainty may affect key moderators of sensorimotor development, such as an individual's affective state, attention, confidence, and participation in physical activity behaviours (Robinson \& Freeston, 2015; Del Popolo Cristaldi et al., 2021). As such, one must not overlook the potential contribution that the construct plays in more applied daily living skills.

From a practical perspective, many autism researchers advocate the provision of explicit contextual information about the underlying statistical properties of a task (e.g., Qian \& Lipkin, 2011; Gomot \& Wicker, 2012; Vermeulen, 2015; Haker et al., 2016). Though clearly beneficial in many settings, our data suggest that this approach is not necessarily appropriate for developing sensorimotor skills that are inherently changeable and unpredictable in nature. Instead, results support strategies that address the implicit, heterogeneous difficulties that many autistic people face when processing dynamic sensory cues. To alleviate these difficulties, practitioners could look to make task environments feel more predictable for autistic people (e.g., through reducing external sensory 'noise', developing individualised routines, or increasing learning repetition blocks; see Haker et al., 2016). Similarly though, practitioners should also look to help individuals deal with volatile and unpredictable elements of sensorimotor skills. Indeed, personalised task modifications may not always be possible at a practical level, and so future work could focus on developing strategies that facilitate the sampling of 'optimal' sensory cues (e.g., see feedforward gaze training: Wilson \& Vine, 2018; environmental scaffolding: Van de Cruys et al., 2014). 
Nevertheless, there are some key limitations that should be considered in future work. Firstly, our results are based on indirect measures of sensorimotor prediction, which were taken from a relatively small number of trials. Studies suggest that changes in prior contextual beliefs can be detected within ten repetitions (Verstynen \& Sabes, 2011), however future investigations may wish to examine longer-term adaptations in predictive sensorimotor control and learning (as in Vossel et al., 2014; Lawson et al., 2017). Moreover, future work could incorporate self-rating methods that directly index participants' trial-by-trial predictions (e.g., Pasturel et al., 2020). Secondly, despite being unconstrained and naturalistic in design, the racquetball task was performed under tightly-controlled VR conditions. Hence, some potentially significant factors that contribute to sensorimotor issues may have been overlooked (e.g., access to support, social/developmental differences). We recommend that future research explores how 'real-world' daily living skills can be enhanced in autistic people, especially in activities that are deemed important or challenging for neurodivergent populations (e.g., driving, occupational skills; see Robledo et al., 2012). Finally, the present study did not conduct any standardised assessments for intellectual disabilities or clinically-related cognitive impairments. It must be stressed here, that the link between these factors and autistic movement abilities is yet to be reliably established (Fournier et al., 2010; Coll et al., 2020) and that our participants reported no co-occurring disabilities that are known to affect sensorimotor control. Furthermore, participants did not display any discernible impairments relating to the understanding of explicit contextual cues in VR, as shown in our Supplementary Information. As such, it seems unlikely that intellectual abilities will have had any meaningfully confounding effects on our observed results.

In sum, the present research found that autism-related sensorimotor difficulties are not alleviated through the provision of explicit contextual cues about environmental uncertainty and stability. Although studied in the unique context of an interceptive VR racquetball task, results indicate that sensorimotor issues are unlikely to reflect any generic impairments in the ability to detect changing environmental probabilities (or any broad intolerance of uncertainty). Instead, results imply that the context-sensitive modulation of top-down predictions and bottom-up sensory cues is atypical in autism. It is therefore recommended that practitioners look to help autistic people build stable action predictions that can help guide movement skills and learning, possibly through the use of inclusive environmental adjustments and/or individualised learning methods. 


\section{References}

Aguinis, H., Gottfredson, R. K., \& Joo, H. (2013). Best-practice recommendations for defining, identifying, and handling outliers. Organizational Research Methods, 16(2), 270-301.

Arthur, T., \& Harris, D. (2021). Predictive eye movements are adjusted in a Bayesoptimal fashion in response to unexpectedly changing environmental probabilities. Cortex, 145, 212-225.

Arthur, T., Harris, D., Buckingham, G., Brosnan, M., Wilson, M., Williams, G., \& Vine, S. (2021). An examination of active inference in autistic adults using immersive virtual reality. Scientific Reports, 11, 20377.

Arthur, T., Vine, S., Brosnan, M., \& Buckingham, G. (2020). Predictive sensorimotor control in autism. Brain, 143(10), 3151-3163.

American Psychological Association. (2013). Diagnostic and statistical manual of mental disorders (DSM-V). Washington DC: American Psychiatric Association.

Balconi, M., Amenta, S., \& Ferrari, C. (2012). Emotional decoding in facial expression, scripts and videos: A comparison between normal, autistic and Asperger children. Research in Autism Spectrum Disorders, 6(1), 193-203.

Balsters, J. H., Apps, M. A., Bolis, D., Lehner, R., Gallagher, L., \& Wenderoth, N. (2017). Disrupted prediction errors index social deficits in autism spectrum disorder. Brain, 140(1), 235-246.

Baron-Cohen, S., Wheelwright, S., Skinner, R., Martin, J., \& Clubley, E. (2001). The autism-spectrum quotient $(A Q)$ : Evidence from asperger syndrome/highfunctioning autism, malesand females, scientists and mathematicians. Journal of Autism and Developmental Disorders, 31(1), 5-17.

Behrens, T. E., Woolrich, M. W., Walton, M. E., \& Rushworth, M. F. (2007). Learning the value of information in an uncertain world. Nature Neuroscience, 10(9), 1214-1221.

Bervoets, J., Milton, D., \& Van de Cruys, S. (2021). Autism and intolerance of uncertainty: an ill-fitting pair. Trends in Cognitive Sciences, 25(12), 1009-1010.

Boulter, C., Freeston, M., South, M., \& Rodgers, J. (2014). Intolerance of uncertainty as a framework for understanding anxiety in children and adolescents with 
autism spectrum disorders. Journal of Autism and Developmental Disorders, 44(6), 1391-1402.

Cannon, J., O'Brien, A. M., Bungert, L., \& Sinha, P. (2021). Prediction in Autism Spectrum Disorder: A Systematic Review of Empirical Evidence. Autism Research, 14(4), 604-630.

Carleton, R. N., Norton, M. P. J., \& Asmundson, G. J. (2007). Fearing the unknown: A short version of the Intolerance of Uncertainty Scale. Journal of Anxiety Disorders, 21(1), 105-117.

Cavallo, A., Romeo, L., Ansuini, C., Battaglia, F., Nobili, L., Pontil, M., . . Becchio, C. (2021). Identifying the signature of prospective motor control in children with autism. Scientific Reports, 11, 3165.

Cesqui, B., Mezzetti, M., Lacquaniti, F., \& d'Avella, A. (2015). Gaze behavior in onehanded catching and its relation with interceptive performance: What the eyes can't tell. PLoS One, 10(3), e0119445.

Chen, L-C., Su, W.-C., Ho, T.-L., Lu, L., Tsai, W.-C., Chiu, Y.-N., \& Jeng, S.-F. (2019). Postural Control and Interceptive Skills in Children With Autism Spectrum Disorder. Physical Therapy, 99(9), 1231-1241.

Coll, S.-M., Foster, N. E., Meilleur, A., Brambati, S. M., \& Hyde, K. L. (2020). Sensorimotor skills in autism spectrum disorder: A meta-analysis. Research in Autism Spectrum Disorders, 76, 101570.

Colombo-Dougovito, A. M., \& Block, M. E. (2019). Fundamental motor skill interventions for children and adolescents on the autism spectrum: a literature review. Review Journal of Autism and Developmental Disorders, 6(2), 159171.

Del Popolo Cristaldi, F., Mento, G., Sarlo, M., \& Buodo, G. (2021). Dealing with uncertainty: A high-density EEG investigation on how intolerance of uncertainty affects emotional predictions. PloS one, 16(7), e0254045.

den Ouden, H. E., Daunizeau, J., Roiser, J., Friston, K. J., \& Stephan, K. E. (2010). Striatal prediction error modulates cortical coupling. Journal of Neuroscience, 30(9), 3210-3219. 
Diaz, G., Cooper, J., \& Hayhoe, M. (2013). Memory and prediction in natural gaze control. Philosophical Transactions of the Royal Society B: Biological Sciences, 368(1628), 20130064.

Dugas, M. J., Freeston, M. H., \& Ladouceur, R. (1997). Intolerance of uncertainty and problem orientation in worry. Cognitive Therapy and Research, 21(6), 593606.

Fabbri-Destro, M., Cattaneo, L., Boria, S., \& Rizzolatti, G. (2009). Planning actions in autism. Experimental Brain Research, 192(3), 521-525.

Faul, F., Erdfelder, E., Lang, A.-G., \& Buchner, A. (2007). G* Power 3: A flexible statistical power analysis program for the social, behavioral, and biomedical sciences. Behavior Research Methods, 39(2), 175-191.

Fournier, K. A., Hass, C. J., Naik, S. K., Lodha, N., \& Cauraugh, J. H. (2010). Motor coordination in autism spectrum disorders: a synthesis and meta-analysis. Journal of Autism and Developmental Disorders, 40(10), 1227-1240.

Franks, I. M., Sanderson, D. J., \& Van Donkelaar, P. (1990). A comparison of directly recorded and derived acceleration data in movement control research. Human Movement Science, 9(6), 573-582.

Friston, K., Lawson, R., \& Frith, C. (2013). On hyperpriors and hypopriors: comment on Pellicano and Burr. Trends in Cognitive Sciences, 17(1).

Friston, K., Samothrakis, S., \& Montague, R. (2012). Active inference and agency: optimal control without cost functions. Biological Cybernetics, 106(8-9), 523541.

Fulceri, F., Tonacci, A., Lucaferro, A., Apicella, F., Narzisi, A., Vincenti, G., . . . Contaldo, A. (2018). Interpersonal motor coordination during joint actions in children with and without autism spectrum disorder: The role of motor information. Research in Developmental Disabilities, 80, 13-23.

Gomot, M., \& Wicker, B. (2012). A challenging, unpredictable world for people with autism spectrum disorder. International Journal of Psychophysiology, 83(2), 240-247.

Gowen, E., \& Hamilton, A. (2013). Motor abilities in autism: a review using a computational context. Journal of Autism and Developmental Disorders, 43(2), 323-344. 
Gowen, E., Vabalas, A., Casson, A. J., \& Poliakoff, E. (2020). Instructions to attend to an observed action increase imitation in autistic adults. Autism, 24(3), 730743.

Gray, R. (2015). The moneyball problem: What is the best way to present situational statistics to an athlete? Paper presented at the 59th International Annual Meeting of the Human Factors and Ergonomics Society, HFES 2014.

Gredin, N. V., Bishop, D. T., Broadbent, D. P., Tucker, A., \& Williams, A. M. (2018). Experts integrate explicit contextual priors and environmental information to improve anticipation efficiency. Journal of Experimental Psychology: Applied, 24(4), 16-24.

Green, D., Baird, G., Barnett, A. L., Henderson, L., Huber, J., \& Henderson, S. E. (2002). The severity and nature of motor impairment in Asperger's syndrome: a comparison with specific developmental disorder of motor function. Journal of Child Psychology and Psychiatry, 43(5), 655-668.

Greene, R. K., Zheng, S., Kinard, J. L., Mosner, M. G., Wiesen, C. A., Kennedy, D. P., \& Dichter, G. S. (2019). Social and nonsocial visual prediction errors in autism spectrum disorder. Autism Research, 12(6), 878-883.

Haker, H., Schneebeli, M., \& Stephan, K. E. (2016). Can Bayesian theories of autism spectrum disorder help improve clinical practice? Frontiers in Psychiatry, 7 , 107.

Hughes, C. (1996). Brief report: Planning problems in autism at the level of motor control. Journal of Autism and Developmental Disorders, 26(1), 99-107.

Jasmin, E., Couture, M., McKinley, P., Reid, G., Fombonne, E., \& Gisel, E. (2009). Sensori-motor and daily living skills of preschool children with autism spectrum disorders. Journal of Autism and Developmental Disorders, 39(2), 231-241.

Kenny, L., Hattersley, C., Molins, B., Buckley, C., Povey, C., \& Pellicano, E. (2016). Which terms should be used to describe autism? Perspectives from the UK autism community. Autism, 20(4), 442-462.

Krassanakis, V., Filippakopoulou, V., \& Nakos, B. (2014). EyeMMV toolbox: An eye movement post-analysis tool based on a two-step spatial dispersion threshold for fixation identification. Journal of Eye Movement Research, 7(1). 
Lawson, R. P., Bisby, J., Nord, C. L., Burgess, N., \& Rees, G. (2021). The Computational, Pharmacological, and Physiological Determinants of Sensory Learning under Uncertainty. Current Biology, 31(1), 163-172.

Lawson, R. P., Mathys, C., \& Rees, G. (2017). Adults with autism overestimate the volatility of the sensory environment. Nature Neuroscience, 20(9), 1293-1299.

Lawson, R. P., Rees, G., \& Friston, K. J. (2014). An aberrant precision account of autism. Frontiers in Human Neuroscience, 8, 302.

Lix, L. M., Keselman, J. C., \& Keselman, H. J. (1996). Consequences of assumption violations revisited: A quantitative review of alternatives to the one-way analysis of variance F test. Review of Educational Research, 66(4), 579-619.

López, B., Donnelly, N., Hadwin, J., \& Leekam, S. (2004). Face processing in highfunctioning adolescents with autism: Evidence for weak central coherence. Visual Cognition, 11(6), 673-688.

MacDonald, M., Lord, C., \& Ulrich, D. (2013). The relationship of motor skills and adaptive behavior skills in young children with autism spectrum disorders. Research in Autism Spectrum Disorders, 7(11), 1383-1390.

Mann, D. L. (2019). Predictive processing in the control of interceptive motor actions. In M. L. Cappuccio (Ed.), Handbook of Embodied Cognition and Sport Psychology (pp. 651-668). Cambridge, Massachusetts The MIT Press.

Mann, D. L., Nakamoto, H., Logt, N., Sikkink, L., \& Brenner, E. (2019). Predictive eye movements when hitting a bouncing ball. Journal of Vision, 19(14), 28.

Manning, C., Kilner, J., Neil, L., Karaminis, T., \& Pellicano, E. (2017). Children on the autism spectrum update their behaviour in response to a volatile environment. Developmental Science, 20(5), 1-13.

Mathys, C. D., Lomakina, E. I., Daunizeau, J., Iglesias, S., Brodersen, K. H., Friston, K. J., \& Stephan, K. E. (2014). Uncertainty in perception and the Hierarchical Gaussian Filter. Frontiers in Human Neuroscience, 8, 825.

McCoy, S. M., Jakicic, J. M., \& Gibbs, B. B. (2016). Comparison of obesity, physical activity, and sedentary behaviors between adolescents with autism spectrum disorders and without. Journal of Autism and Developmental Disorders, 46(7), 2317-2326. 
Navia, J. A., van der Kamp, J., \& Ruiz, L. M. (2013). On the use of situation and body information in goalkeeper actions during a soccer penalty kick. International Journal of Sport Psychology, 44(3), 234-251.

Palacios, E. R., Houghton, C., \& Chadderton, P. (2021). Accounting for uncertainty: inhibition for neural inference in the cerebellum. Proceedings of the Royal Society B, 288(1947), 20210276.

Palmer, C. J., Lawson, R. P., \& Hohwy, J. (2017). Bayesian approaches to autism: Towards volatility, action, and behavior. Psychological Bulletin, 143(5), 521.

Palmer, C. J., Paton, B., Kirkovski, M., Enticott, P. G., \& Hohwy, J. (2015). Context sensitivity in action decreases along the autism spectrum: a predictive processing perspective. Proceedings of the Royal Society B: Biological sciences, 282, 1802.

Pasturel, C., Montagnini, A., \& Perrinet, L. U. (2020). Humans adapt their anticipatory eye movements to the volatility of visual motion properties. PLoS Computational Biology, 16(4), e1007438.

Pickard, H., Hirsch, C., Simonoff, E., \& Happé, F. (2020). Exploring the cognitive, emotional and sensory correlates of social anxiety in autistic and neurotypical adolescents. Journal of Child Psychology and Psychiatry, 61(12), 1317-1327.

Plaisted, K., Swettenham, J., \& Rees, L. (1999). Children with autism show local precedence in a divided attention task and global precedence in a selective attention task. The Journal of Child Psychology and Psychiatry and Allied Disciplines, 40(5), 733-742.

Qian, N., \& Lipkin, R. M. (2011). A learning-style theory for understanding autistic behaviors. Frontiers in Human Neuroscience, 5, 77.

Quattrocki, E., \& Friston, K. (2014). Autism, oxytocin and interoception. Neuroscience \& Biobehavioral Reviews, 47, 410-430.

Robinson, G., \& Freeston, M. (2015). Intolerance of uncertainty as a predictor of performance anxiety and robustness of sport confidence in university studentathletes. Journal of Clinical Sport Psychology, 9(4), 335-344.

Robledo, J., Donnellan, A. M., \& Strandt-Conroy, K. (2012). An exploration of sensory and movement differences from the perspective of individuals with autism. Frontiers in Integrative Neuroscience, 6, 107. 
Ruzich, E., Allison, C., Smith, P., Watson, P., Auyeung, B., Ring, H., \& Baron-Cohen, S. (2015). Measuring autistic traits in the general population: a systematic review of the Autism-Spectrum Quotient (AQ) in a nonclinical population sample of 6,900 typical adult males and females. Molecular Autism, 6(2), 1-12. Sapey-Triomphe, L.-A., Weilnhammer, V. A., \& Wagemans, J. (2021). Associative learning under uncertainty in adults with autism: Intact learning of the cueoutcome contingency, but slower updating of priors. Autism, 17, 13623613211045026.

Schmitz, C., Martineau, J., Barthélémy, C., \& Assaiante, C. (2003). Motor control and children with autism: deficit of anticipatory function? Neuroscience Letters, 348(1), 17-20.

Soroor, G., Mokhtari, S., \& Pouretemad, H. (2021). Priming Global Processing Strategy Improves the Perceptual Performance of Children with Autism Spectrum Disorders. Journal of Autism and Developmental Disorders, https://doi.org/10.1007/s10803-10021-05007-10807.

Sutera, S., Pandey, J., Esser, E. L., Rosenthal, M. A., Wilson, L. B., Barton, M., . . . Dumont-Mathieu, T. (2007). Predictors of optimal outcome in toddlers diagnosed with autism spectrum disorders. Journal of Autism and Developmental Disorders, 37(1), 98-107.

Thillay, A., Lemaire, M., Roux, S., Houy-Durand, E., Barthélémy, C., Knight, R. T., . . . Bonnet-Brilhault, F. (2016). Atypical brain mechanisms of prediction according to uncertainty in autism. Frontiers in Neuroscience, 10, 317.

Van de Cruys, S., Evers, K., Van der Hallen, R., Van Eylen, L., Boets, B., de-Wit, L., \& Wagemans, J. (2014). Precise minds in uncertain worlds: Predictive coding in autism. Psychological Review, 121(4), 649.

Vasa, R. A., Kreiser, N. L., Keefer, A., Singh, V., \& Mostofsky, S. H. (2018). Relationships between autism spectrum disorder and intolerance of uncertainty. Autism Research, 11(4), 636-644.

Vermeulen, P. (2015). Context blindness in autism spectrum disorder: Not using the forest to see the trees as trees. Focus on Autism and Other Developmental Disabilities, 30(3), 182-192. 
Verstynen, T., \& Sabes, P. N. (2011). How each movement changes the next: an experimental and theoretical study of fast adaptive priors in reaching. Journal of Neuroscience, 31(27), 10050-10059.

Vossel, S., Mathys, C., Daunizeau, J., Bauer, M., Driver, J., Friston, K. J., \& Stephan, K. E. (2014). Spatial attention, precision, and Bayesian inference: a study of saccadic response speed. Cerebral Cortex, 24(6), 1436-1450.

Whyatt, C., \& Craig, C. (2013). Interceptive skills in children aged 9-11 years, diagnosed with Autism Spectrum Disorder. Research in Autism Spectrum Disorders, 7(5), 613-623.

Wigham, S., Rodgers, J., South, M., McConachie, H., \& Freeston, M. (2015). The interplay between sensory processing abnormalities, intolerance of uncertainty, anxiety and restricted and repetitive behaviours in autism spectrum disorder. Journal of Autism and Developmental Disorders, 45(4), 943-952.

Wilson, M. R., \& Vine, S. J. (2018). The use of gaze training to expedite motor skill acquisition. In Handbook of Sport Neuroscience and Psychophysiology (pp. 237-247): Routledge.

Woodbury-Smith, M. R., Robinson, J., Wheelwright, S., \& Baron-Cohen, S. (2005). Screening adults for Asperger syndrome using the AQ: A preliminary study of its diagnostic validity in clinical practice. Journal of Autism and Developmental Disorders, 35(3), 331-335.

World Health Organisation. (2012). The ICD-10 Classification of Mental and Behavioural Disoders - Clinical Descriptions and Diagnostic Guidelines. . Geneva: WHO.

Yon, D., \& Frith, C. D. (2021). Precision and the Bayesian brain. Current Biology, 31(17), R1026-R1032.

Yu, A., \& Dayan, P. (2003). Uncertainty, neuromodulation, and attention. Neuron, 46, 681-692. 


\section{Supplementary Information}

\section{Exploratory Analysis of Gaze Fixation Data}

Follow-up tests explored whether group-dependent changes in gaze behaviours derive from altered volatility processing or whether they are simply reflecting participants' ability to use prior probabilistic information. For instance, the cued block contained periods of 6-12 trials where the likelihood of facing a bouncy ball was described as 'high' to participants. Thus, it is possible that higher pitch averages in cued versus control trials are being driven by data from these specific datapoints. Conversely, autistic participants may have difficulties interpreting these cues, leading to nonsignificant changes between conditions. In the analysis that follows, data from selected trials are scrutinised within each condition, with two participants and their matched counterparts excluded due to missing outcome values (remaining $n=40$ ).

Firstly, gaze data from trials that immediately followed a 'high' probabilistic cue were extracted for each participant. Prior to these trials, participants viewed a 'hawkeye' illustration which projected an equal probability of facing a normal or bouncy ball (see Figure 1). This was accompanied by an indication that the current likelihood of facing a bouncy ball was relatively 'high'. If these probabilistic cues were being readily used by participants, then one would expect subsequent predictive bounce fixations to be higher than control values. Indeed, a mixed-model ANOVA found a significant effect of condition on the extracted fixation data $\left(F(1,38)=50.15, p<.001, \eta_{p}^{2}=0.57 ; \mathrm{BF}_{10}=\right.$ $\left.2.67 \times 10^{5}\right)$, with both groups exhibiting increases in the height of their predictive gaze fixations (Wilcoxon Signed-Rank: $Z=5.51, p<.001, \mathrm{BF}_{10}=4.76 \times 10^{5}$ ). Importantly, no significant interactions were recorded $\left(F(1,38)=2.99, p=.09, \eta_{p}^{2}=0.07 ; B F_{10}=0.91\right)$.

Next, mixed-model ANOVAs were repeated using data from catch trials only. In these trials, no prior information about likely ball bounciness was provided; participants were simply cued that the environment had changed. Notably, the significant interaction effects observed in our main analyses were replicated in this data $(F(1,38)=5.24, p=$ $\left..03, \eta_{p}^{2}=0.12\right)$, albeit with weaker statistical strength $\left(\mathrm{BF}_{10}=2.40\right)$. Together, results suggest that group-dependent changes in visual sampling behaviour were not due to differences in the interpretation or understanding of our explicit VR cues. Instead, they appear related to expectations about environmental stability (see main discussion). 


\section{Figure Captions}

Figure 1. The Virtual Racquetball task. Participants were required to intercept balls that bounced with either normal or unexpectedly-high levels of elasticity. Gameplay footage of the virtual racquet, ball, and target is illustrated in panel A. During control trials, participants received no explicit information about levels of ball bounciness and changing task conditions. However, three additional pieces of information were provided ahead of cued trials. Firstly, changes in task conditions were signalled using 'game level' transitions (B), which notified participants that they were about to enter a new environmental context. The proportion of normal and bouncy balls in each level were then projected in space using visual 'hawkeye' cues (C). A simulated 'bounceometer' on the front wall (shown in D) also confirmed whether a bouncy ball was 'low', 'medium', or 'high' in likelihood ahead of each trial. Together, these cues explicitly informed participants about dynamic task probabilities and environmental volatility. Supplementary Videos of the protocol can be found at: https://osf.io/5y48g/.

Figure 2. Schematic Illustration of the Experimental Protocol. Participants were presented with a series of balls that bounced with either normal (blue circles) or unexpectedly-high (red circles) levels of elasticity. Though trial order sequences were the same in each condition, Cued trials were separated into six game levels. Upon entering a new game level, participants received projected 'hawkeye' cues (see arrows). Each subsequent trial was then preceded by a visual indicator, which stated whether the likelihood of facing a bouncy ball was 'low' $(17 \%)$, 'medium' (33\%), or 'high' (50\%) for this level. Conversely, balls in the non-cued condition were presented as one continuous sequence of trials, with no additional visual information. *Note that both blocks ended with nine catch trials that contained no explicit probabilistic cues.

Figure 3. Task Performance. The proportion of balls successfully intercepted in control and cued conditions for each group. NT: neurotypical; ASD: autism spectrum disorder.

Figure 4. Average peak hand velocities $(\mathbf{A})$ and range of motion (C) during foreswing actions. Between-condition changes are illustrated in panels B and D. NT: neurotypical; ASD: autism spectrum disorder; * denotes significant difference $(p<.05)$.

Figure 5. The average onset times $(\mathbf{A})$, durations $(\mathbf{C})$, and pitch angle $(\mathbf{E})$ of predictive bounce fixations. Between-condition changes are illustrated in panels B, D, and F. NT: neurotypical; ASD: autism spectrum disorder; ${ }^{*}$ denotes significant differences $(p<.05)$.

Figure 6. Trial-by-trial standard deviations in the pitch angle of predictive bounce fixations during control and cued blocks. NT: neurotypical; ASD: autism spectrum disorder; * denotes significant differences $(p<.05)$. 


\section{Figures}

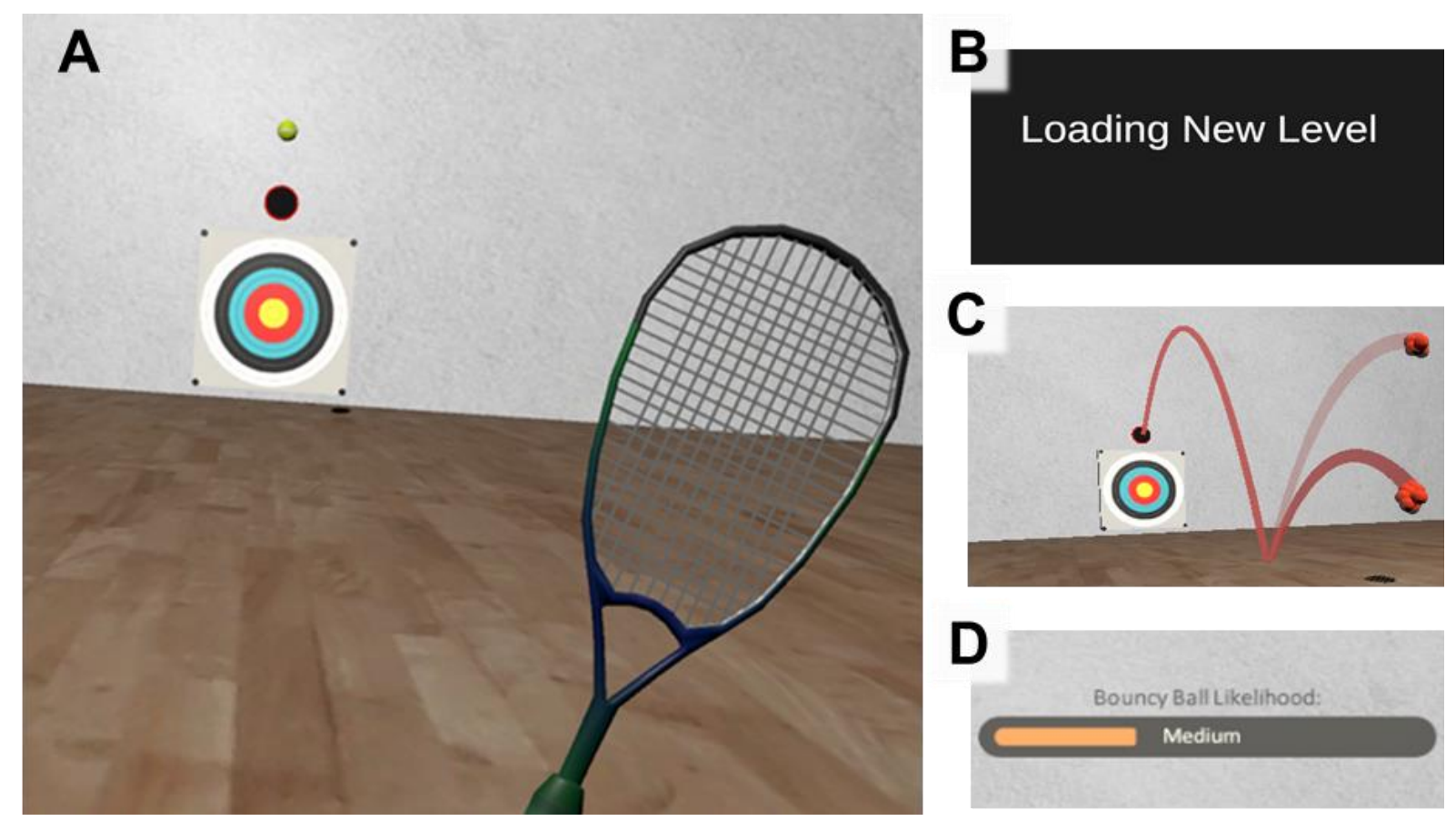

Figure 1. The Virtual Racquetball task. Participants were required to intercept balls that bounced with either normal or unexpectedly-high levels of elasticity. Gameplay footage of the virtual racquet, ball, and target is illustrated in panel A. During control trials, participants received no explicit information about levels of ball bounciness and changing task conditions. However, three additional pieces of information were provided ahead of cued trials. Firstly, changes in task conditions were signalled using 'game level' transitions (B), which notified participants that they were about to enter a new environmental context. The proportion of normal and bouncy balls in each level were then projected in space using visual 'hawkeye' cues (C). A simulated 'bounceometer' on the front wall (shown in D) also confirmed whether a bouncy ball was 'low', 'medium', or 'high' in likelihood ahead of each trial. Together, these cues explicitly informed participants about dynamic task probabilities and environmental volatility. Supplementary Videos of the protocol can be found at: https://osf.io/5y48g/. 
A

\section{Non-Cued Block}

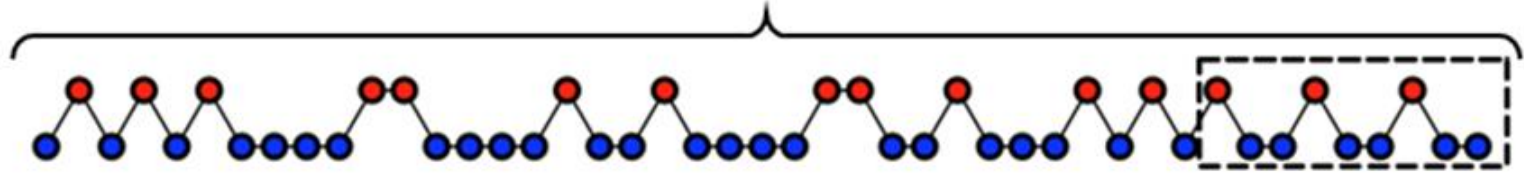

Catch Trials

B

Cued Block

Level One Level Two

Level Three

Level Four Level Five

Level Six*

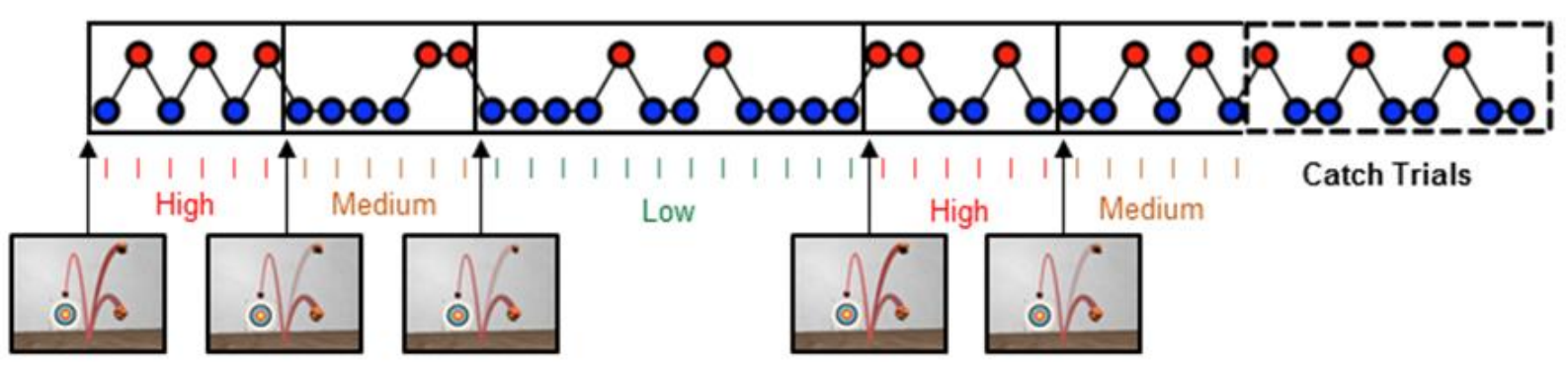

Figure 2. Schematic Illustration of the Experimental Protocol. Participants were presented with a series of balls that bounced with either normal (blue circles) or unexpectedly-high (red circles) levels of elasticity. Though trial order sequences were the same in each condition, Cued trials were separated into six game levels. Upon entering a new game level, participants received projected 'hawkeye' cues (see arrows). Each subsequent trial was then preceded by a visual indicator, which stated whether the likelihood of facing a bouncy ball was 'low' (17\%), 'medium' (33\%), or 'high' (50\%) for this level. Conversely, balls in the non-cued condition were presented as one continuous sequence of trials, with no additional visual information. *Note that both blocks ended with nine catch trials that contained no explicit probabilistic cues. 
Control Conditions

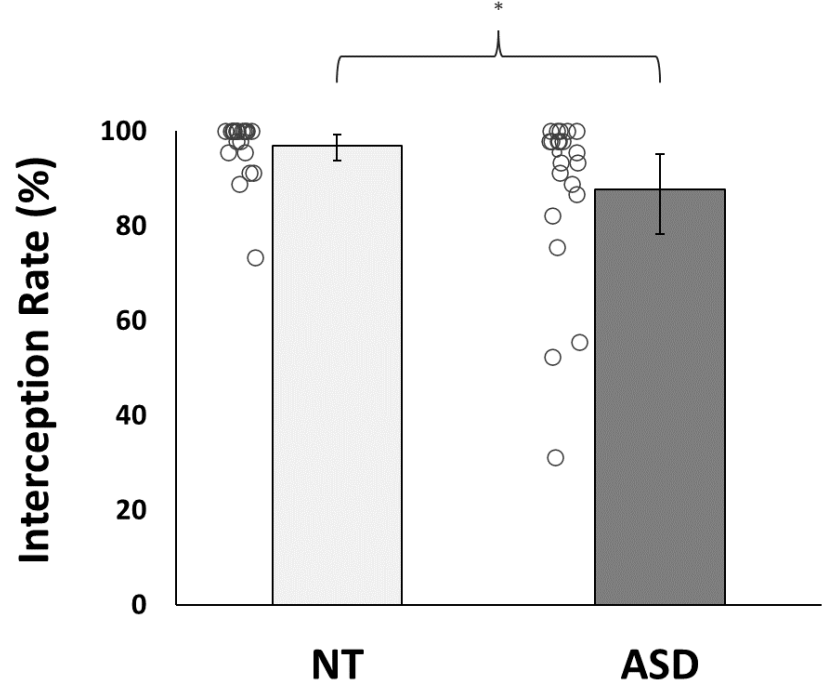

Cued Conditions

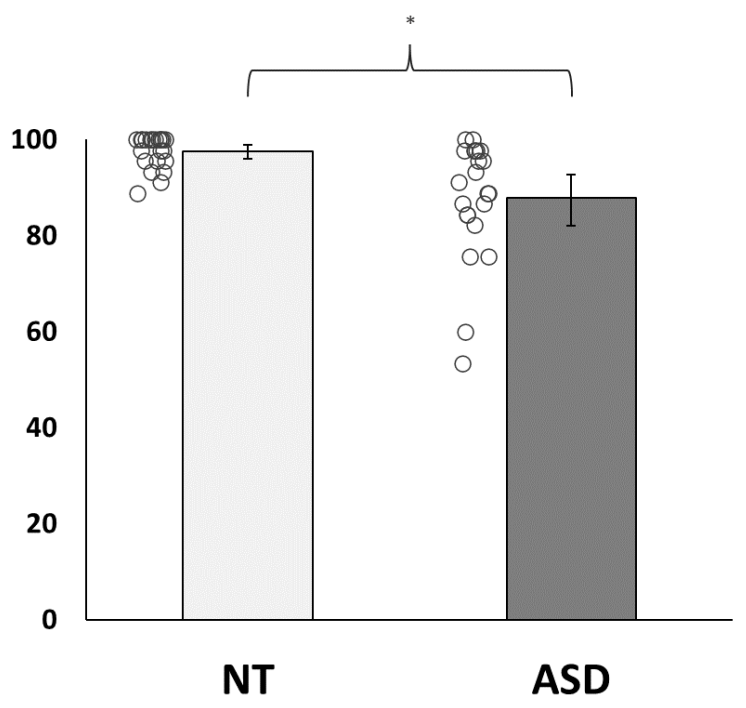

Figure 3. Task Performance. The proportion of balls successfully intercepted in control and cued conditions for each group. NT: neurotypical; ASD: autism spectrum disorder. Error bars indicate Bias-Corrected Accelerated bootstrapped 95\% confidence intervals (based on 10000 samples). *denotes significant between-group difference $(p<.05)$. 
A

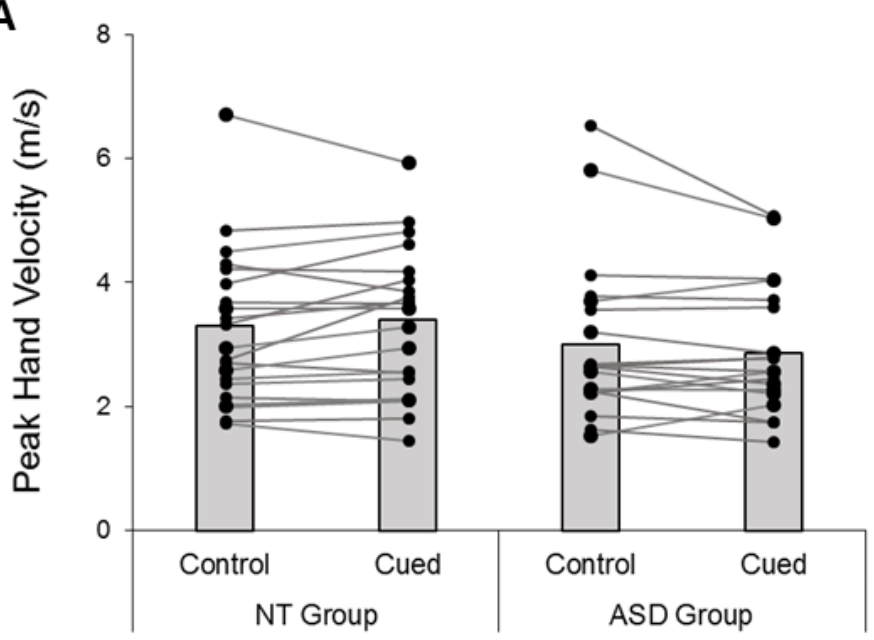

C

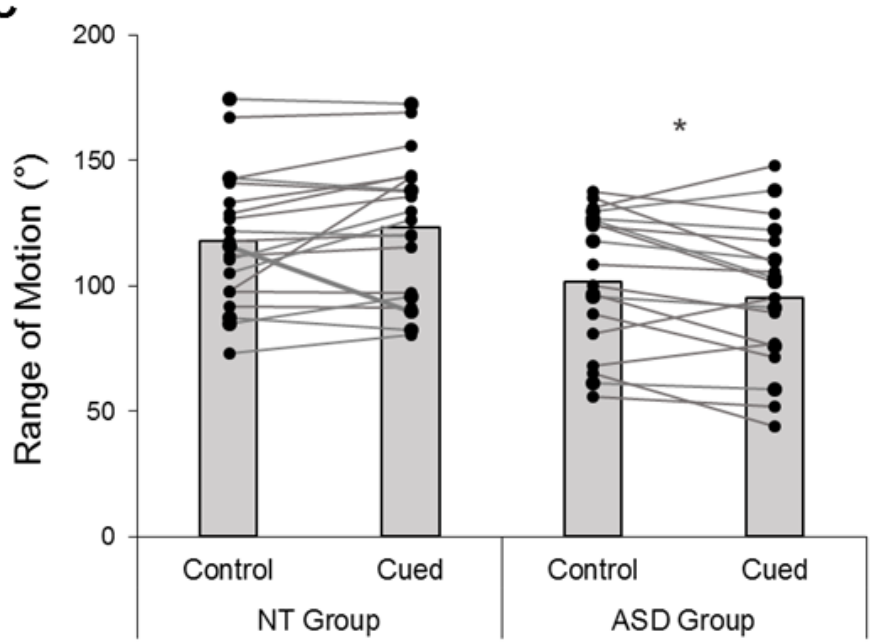

B

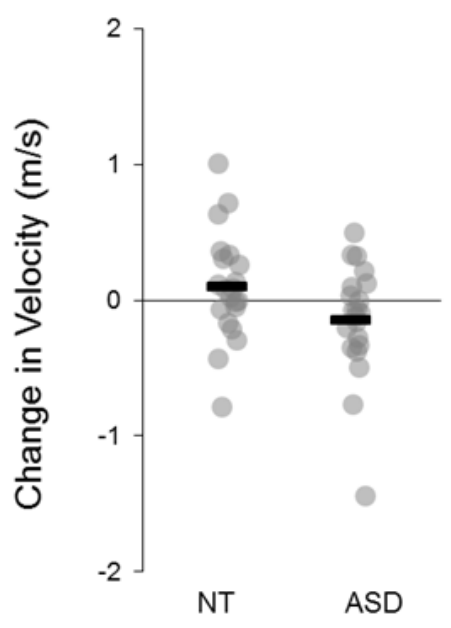

D

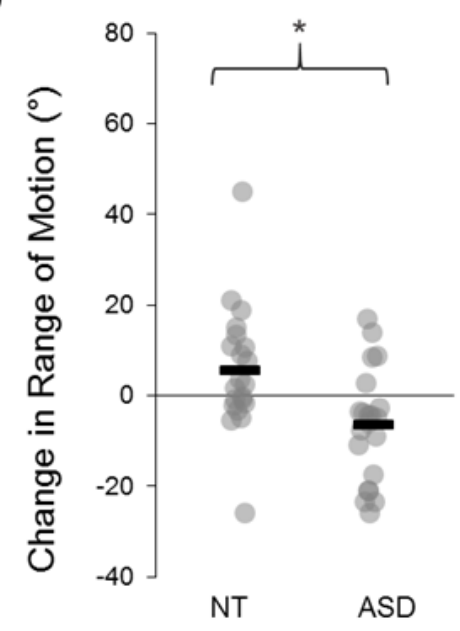

Figure 4. Average peak hand velocities $(\mathbf{A})$ and range of motion $(\mathbf{C})$ during foreswing actions. Between-condition changes are illustrated in panels B and D. NT: neurotypical; ASD: autism spectrum disorder; ${ }^{*}$ denotes significant difference $(p<.05)$. 
A

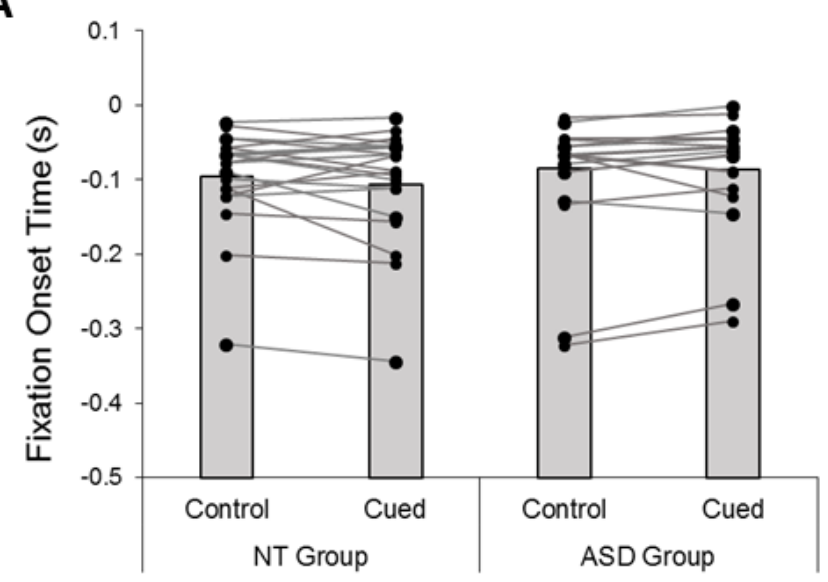

C

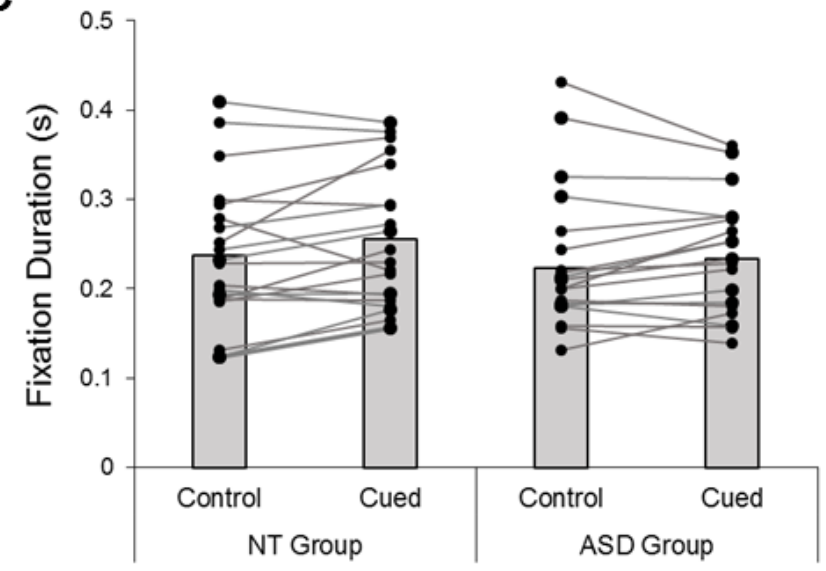

E

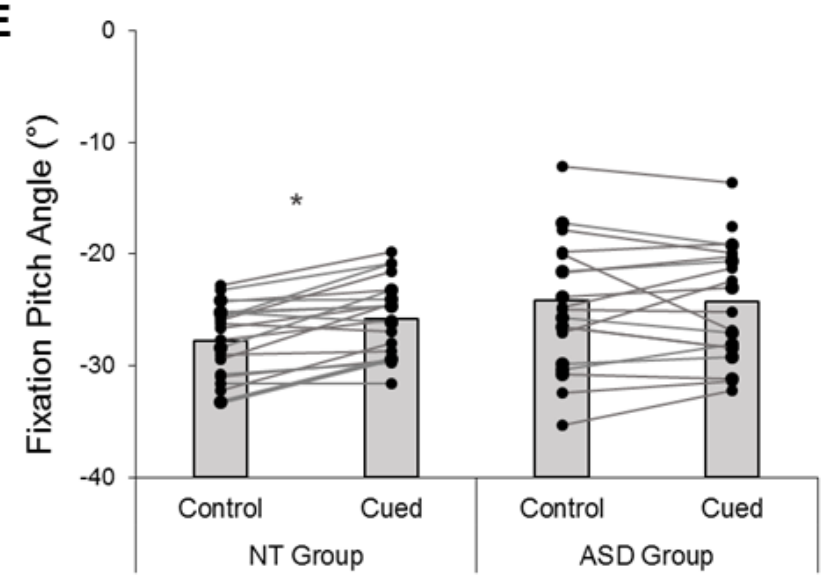

B
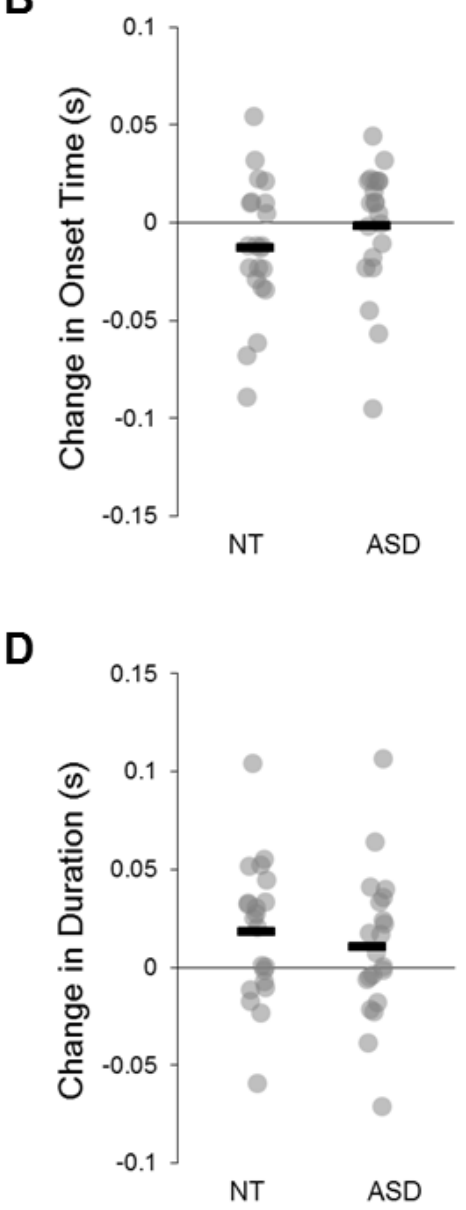

$\mathbf{F}$

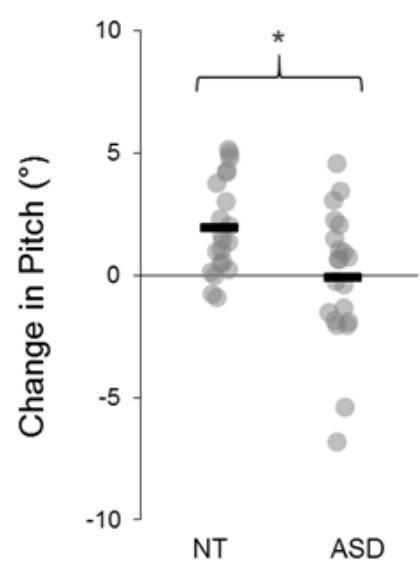

Figure 5. The average onset times (A), durations (C), and pitch angle (E) of predictive bounce fixations. Between-condition changes are illustrated in panels $\mathbf{B}, \mathbf{D}$, and F. NT: neurotypical; ASD: autism spectrum disorder; ${ }^{*}$ denotes significant differences $(p<.05)$. 
Control Conditions

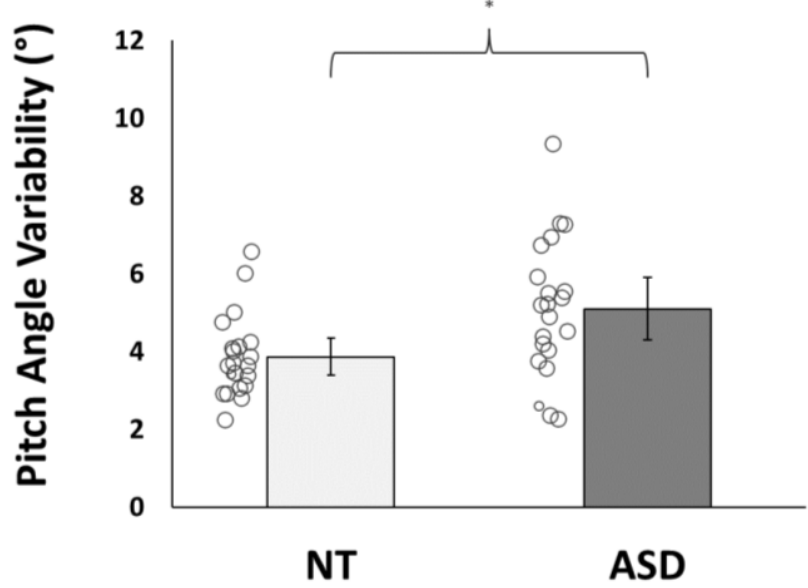

Cued Conditions

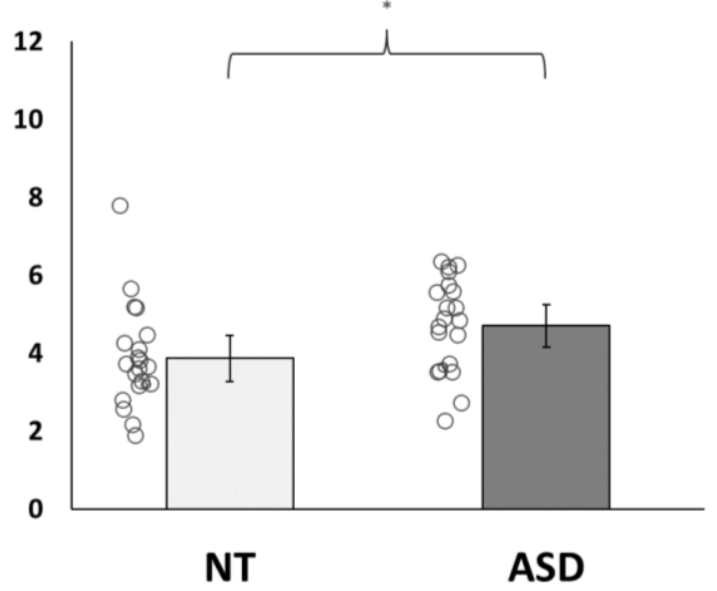

Figure 6. Trial-by-trial standard deviations in the pitch angle of predictive bounce fixations during control and cued blocks. NT: neurotypical; ASD: autism spectrum disorder; Error bars indicate $95 \%$ confidence intervals. ${ }^{*}$ denotes significant between-group difference $(p<.05)$. 


\section{Tables}

Table 1. Participant Characteristics. Group averages (and standard deviations) for all demographics and self-report measures that were recorded in the study.

\begin{tabular}{lcc}
\hline & ASD Group & NT Group \\
\hline Sample Size & $n=22$ & $n=22$ \\
Age & $29.05(7.52)$ years & $29.55(6.75)$ years \\
Gender & 15 male, 7 female & 15 male, 7 female \\
Dominant Hand & 21 right, 1 left & 21 right, 1 left \\
AQ Total Score & $35.86(5.37)$ & $15.59(7.96)$ \\
IUS-S Score $^{*}$ & $38.86(9.98)$ & $27.00(10.14)$ \\
\hline
\end{tabular}

ASD: autism spectrum disorder; NT: neurotypical, AQ: 50-item Autistic Quotient, IUS-S: Intolerance of Uncertainty Scale - shortened version; *denotes significant between-group difference $(p<.001)$. 